\title{
Radiation Damage and Annealing in 1310nm InGaAsP/InP Lasers for the CMS Tracker
}

\author{
K. Gill, G. Cervelli, R. Grabit, F. Jensen, and F. Vasey. \\ EP Division, CERN, CH-1211, Geneva 23, Switzerland.
}

\begin{abstract}
Radiation damage in $1310 \mathrm{~nm}$ InGaAsP/InP multi-quantum-well lasers caused by $0.8 \mathrm{MeV}$ neutrons is compared with the damage from other radiation sources, in terms of the increase in laser threshold current. The annealing behavior is then presented both in terms of both temperature and forward-bias current dependence. The annealing can be described by a model where radiation induced defects have a uniform distribution of activation energies for annealing. This model can then be used to predict the long-term damage expected for lasers operating inside the CMS tracker.
\end{abstract}

Keywords: Optical link, laser, radiation damage, annealing, high energy physics

\section{INTRODUCTION}

A system of 50000 analogue optical links, using InGaAsP/InP lasers operating at 1310nm, will be used to transfer data signals from more than 10 million microstrip detector channels in the tracker system of the Compact Muon Solenoid (CMS) experiment[1-3]. This experiment is currently under construction and will operate at the Large Hadron Collider (LHC), a 7+7 TeV proton-proton collider also being built at CERN. Radiation damage is a major concern in the LHC experiments, especially for component situated in tracking detectors near the beam interaction points. For example, lasers and other optical link components operating inside the CMS tracker will be exposed to more than $10^{14}$ particles $/ \mathrm{cm}^{2}$ and ionizing doses $\sim 100 \mathrm{kGy}[2,3]$ over the 10 year operating lifetime.

Radiation damage effects observed in semiconductor lasers are caused by displacement or ionization mechanisms[4,5]. At the level of the laser die, the effects of displacement damage are usually more important than those of ionization damage, at least for the radiation levels expected inside the CMS tracker[6,7]. Displacement damage causes host atoms to be removed from their initial crystal lattice positions in collisions with incident energetic particles or other recoiling atoms[4]. Vacancy and interstitial related defects are created that may introduce energy levels in the band-gap. These defects can act as nonradiative recombination centers, causing the threshold current in lasers to increase and the output efficiency to decrease[4,6]. Some fraction of the damage generally recovers, or anneals, in the time following irradiation[6].

All the optical link component types that will be used in the CMS tracker (InGaAsP/InP lasers, InGaAs p-i-n photodiodes, single-mode optical fibers and optical connectors) have been tested[6-11] with ${ }^{60} \mathrm{Co}$ photons and at least one heavy particle source, including 6MeV (average energy) neutrons (at ISN/SARA, Grenoble), 330MeV pions (at PSI, Villigen), and 24GeV protons (at CERN). These sources were chosen as the particle species and energies broadly represent the overall radiation spectrum that will be encountered inside the CMS Tracker[2,3].

In this paper we focus on one type of $1310 \mathrm{~nm}$ InGaAsP/InP laser, first of all comparing the radiation damage due to a fifth radiation source, $0.8 \mathrm{MeV}$ (average energy) neutrons from the Prospero reactor at CEA Valduc, France[12], with that from the other radiation sources. The second part of the experimental work was to measure the annealing behavior following $0.8 \mathrm{MeV}$ neutron irradiation as both a function of temperature and bias-current. Finally a model is developed to describe the annealing behavior in terms of defect activation energies. Based on the results for the relative damage of $0.8 \mathrm{MeV}$ neutrons compared to the other sources, and the predictions of the model for longer-term annealing, a simulation of the damage expected for lasers inside the CMS tracker is then outlined.

\footnotetext{
* Corresponding author.

E-mail: karl.gill@ cern.ch, telephone: (+41) 22 7678583, fax: (+41) 227672800
} 


\subsection{Devices}

\section{EXPERIMENT}

The components under study were NEC 1310nm edge-emitting InGaAsP/InP MQW (multi-quantum well) DCPBH (double channel planar buried heterostructure[13]) lasers, mounted on Si-submounts in compact mini-DIL packages with singlemode fiber pigtails, supplied by Italtel. There were no components included in the package, such as lenses, that could be degraded by ionization damage[14]. The initial laser threshold currents were 8-13mA and the output efficiencies (out of the fibre) were $30-70 \mu \mathrm{W} / \mathrm{mA}$ at $20^{\circ} \mathrm{C}$.

\subsection{Irradiation and annealing procedures}

The overall investigation was divided into 3 irradiation tests (A, B and C) outlined in Table 1.

Test A was a measurement of damage and annealing with $0.8 \mathrm{MeV}$ neutrons, under similar conditions to previous tests made at other radiation sources. The effect of $0.8 \mathrm{MeV}$ neutron damage was then compared with the earlier measurements from other sources[8].

Test $\mathrm{B}$ measured the rate of annealing at different temperatures in lasers irradiated with $0.8 \mathrm{MeV}$ neutrons. The aim was to determine the activation energies for annealing using an approach similar to that of Shaw et al.[15] where annealing was studied for irradiated InGaAs photodiodes.

Test $\mathrm{C}$ was then carried out to determine how much the annealing rate could be accelerated by applying forward bias. The effect of bias-enhanced annealing in irradiated $\mathrm{AlGaAs} / \mathrm{GaAs}$ lasers was recently reported by Zhao et al.[13]. A significant acceleration of annealing in InGaAsP/InP lasers would have important consequences for lasers used inside the CMS tracker, such that the long term accumulation of radiation damage might be suppressed by applying larger dc bias currents.

In all 3 tests the lasers were connected both optically and electrically in order to measure the damage and annealing effects in-situ. In this paper we will consider only the damage to the laser threshold current. This parameter is observed to be degraded much more by radiation damage than the efficiency[6]. Moreover, our previous radiation damage measurements have shown that, in these particular lasers, the degradation of the efficiency by displacement damage is proportional to the threshold current increase[6].

The reactor power, and therefore the neutron flux, was constant throughout each irradiation. Indium foil dosimeters were placed close to the devices and fluences were determined with $10 \%$ uncertainty. The ionizing dose due to gamma rays emitted by the reactor was $\sim 750$ Gy per $10^{14} \mathrm{n} / \mathrm{cm}^{2}$. Previous measurements[7] have shown that there is no significant damage to this type of laser after $100 \mathrm{kGy}$ dose, therefore this background gamma dose will not have caused any additional damage in the lasers.

Table 1: Experimental conditions for the radiation damage and annealing studies.

\begin{tabular}{|c|c|c|c|c|c|c|}
\hline Test & $\begin{array}{l}\text { No. } \\
\text { lasers }\end{array}$ & $\begin{array}{c}\text { Fluence } \\
0.8 \mathrm{MeV} \text { neutrons } \\
10^{14} \mathrm{n} / \mathrm{cm}^{2}( \pm 10 \%)\end{array}$ & $\begin{array}{l}\text { Irrad } \\
\text { time } \\
\text { (hrs) }\end{array}$ & $\begin{array}{l}\text { Annealing } \\
\text { time } \\
\text { (hrs) }\end{array}$ & $\begin{array}{l}\text { Irradiation conditions } \\
\text { (bias and temperature) }\end{array}$ & $\begin{array}{l}\text { Annealing conditions } \\
\text { (bias and temperature) }\end{array}$ \\
\hline A & 4 & 10.5 to 11.0 & 6.5 & 115 & $\begin{array}{l}\text { Biased at } 5 \text { to } 10 \mathrm{~mA} \\
\text { above threshold, } \\
\text { Ambient } \mathrm{T}\left(20-30^{\circ} \mathrm{C}\right)\end{array}$ & $\begin{array}{l}\text { Biased at } 5 \text { to } 10 \mathrm{~mA} \\
\text { above threshold, } \\
\text { Ambient } \mathrm{T}\left(20-30^{\circ} \mathrm{C}\right)\end{array}$ \\
\hline B & 12 & 9.6 to 11.5 & 6.3 & $\begin{array}{l}16\left(\text { at }-13^{\circ} \mathrm{C}\right) \\
310 \text { (at other } \\
\text { temperatures) }\end{array}$ & $\begin{array}{c}\text { Unbiased, } \\
\text { Cooled }\left(-12^{\circ} \mathrm{C}\right)\end{array}$ & $\begin{array}{c}\text { Unbiased, } \\
\text { Heated }\left(\mathrm{T}=20,40,60,80^{\circ} \mathrm{C},\right. \\
3 \text { devices per } \mathrm{T} \text { value })\end{array}$ \\
\hline $\mathrm{C}$ & 8 & 8.1 to 11.2 & 6.5 & 115 & $\begin{array}{c}\text { Unbiased, } \\
\text { Ambient } \mathrm{T}\left(20-30^{\circ} \mathrm{C}\right)\end{array}$ & $\begin{array}{c}\text { Biased }(0,60,80,100 \mathrm{~mA}, \\
2 \text { devices per current value) } \\
\text { Ambient } \mathrm{T}\left(20-30^{\circ} \mathrm{C}\right)\end{array}$ \\
\hline
\end{tabular}




\section{RESULTS}

\subsection{Test A - Relative Damage Factor for 0.8MeV Neutrons}

The laser threshold increase versus neutron fluence is shown in Fig. 1. The damage is approximately linear with fluence. There is some decrease in the rate of damage with increasing fluence, which is a typical effect of annealing taking place during the irradiation period.

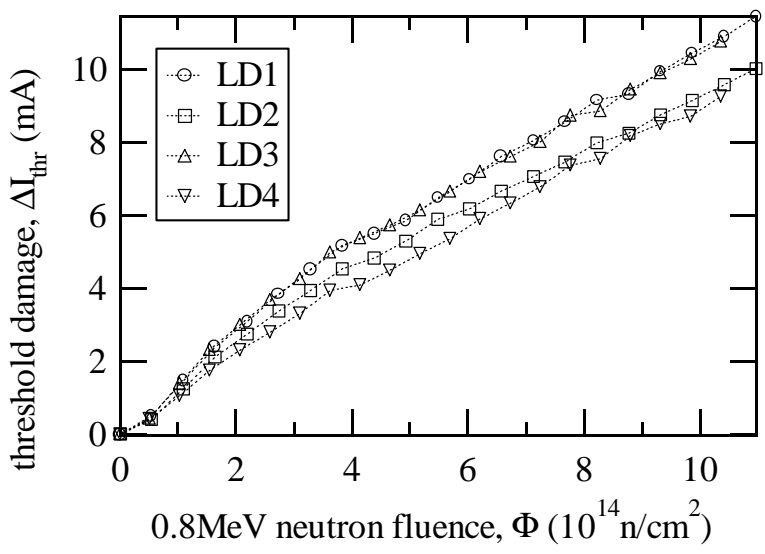

Fig. 1: Threshold current increase vs $0.8 \mathrm{MeV}$ neutron fluence.

Our previous comparisons between the damage from different sources have been made based on a 96 hour irradiation period and a total particle fluence of $5 \times 10^{14} / \mathrm{cm}^{2}[8]$. The new data therefore have to be normalized to this lower fluence, whilst also taking into account the extra annealing that would occur during the longer irradiation period. This re-scaling was done by considering the effect of 15 consecutive 6.5 hour irradiation steps, taking into account the annealing that occurs for the damage already introduced during the earlier steps. (The $15^{\text {th }}$ step is truncated by $1.5 \mathrm{hrs}$ to arrive at 96 hours exposure.)

Fig. 2 compares the average damage of $0.8 \mathrm{MeV}$ neutrons with that from other radiation sources[8]. The displacement damage factors for $0.8 \mathrm{MeV}$ neutrons relative to $6 \mathrm{MeV}$ (average energy) neutrons, $330 \mathrm{MeV}$ pions and $24 \mathrm{GeV}$ protons are $1 / 3.1,1 / 11.5$ and $1 / 8.4$ respectively, with an uncertainty of $\sim 10 \%$ in each case.

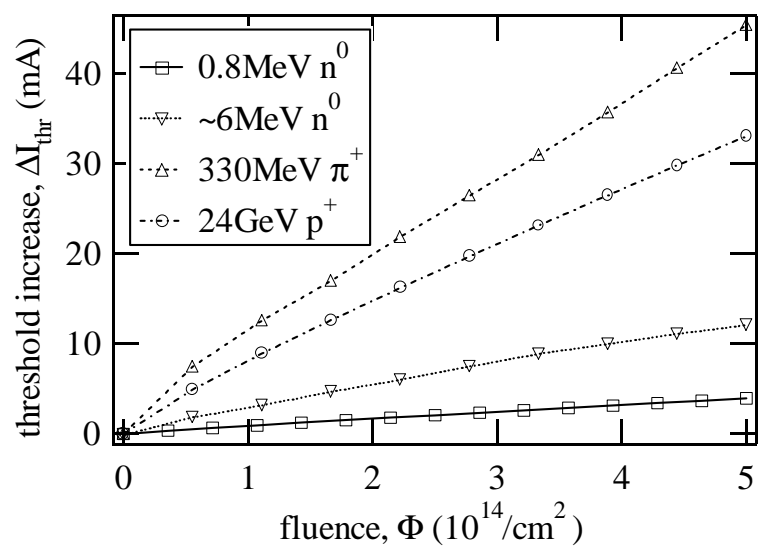

Fig. 2: Average threshold current increase due to $0.8 \mathrm{MeV}$ neutron damage, compared with $330 \mathrm{MeV}$ pion, $24 \mathrm{GeV}$ proton and $\sim 6 \mathrm{MeV}$ neutron damage. The data have been scaled to a fluence of $5 \times 10^{14} / \mathrm{cm}^{2}$ in a $96 \mathrm{hr}$ irradiation period. 


\subsection{Test B - Annealing vs Temperature}

The annealing behavior was studied in Test B using the same type of InGaAsP/InP lasers irradiated with $0.8 \mathrm{MeV}$ neutrons at Valduc. 12 devices were irradiated and annealed at approximately $-13^{\circ} \mathrm{C}$ (without electrical bias). Fig. 3 shows the damage and annealing of the laser threshold currents, along with the temperature of the samples. The fluence received was approximately uniform over all the devices at $10.5 \times 10^{15} \mathrm{n} / \mathrm{cm}^{2}( \pm 10 \%)$. The threshold increase was around $9 \mathrm{~mA}$ in all the lasers and $15 \%$ of this damage annealed in the 16 hours following irradiation. The lasers were then stored (electricall shorted) at $-35^{\circ} \mathrm{C}$ in order to suppress any further annealing during the time between the end of the Valduc measurements and the start of the measurements in the oven at CERN.

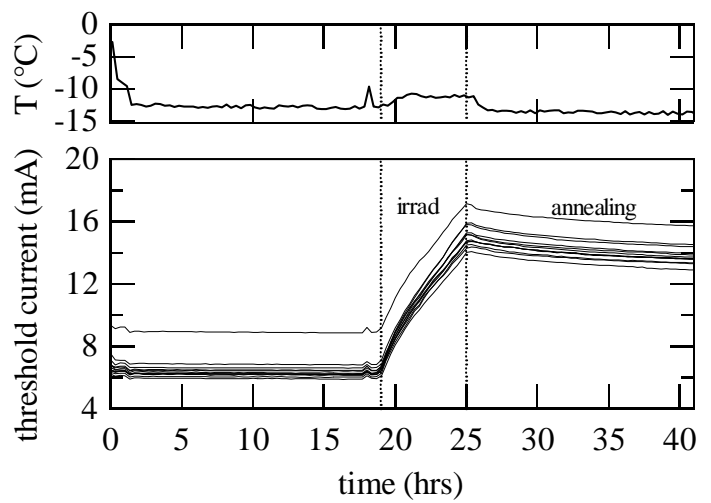

Fig. 3: Laser threshold current before, during and after irradiation at approximately $-13^{\circ} \mathrm{C}$.

Four groups of 3 irradiated devices were then annealed for 310 hours at $20,40,60$, and $80^{\circ} \mathrm{C}$ respectively. The annealing of the radiation induced defects in the lasers was measured in terms of the change in the laser threshold current, such that the fraction of unannealed defects is assumed to be directly proportional to the remaining fraction of threshold damage. In Fig. 4 the annealing is shown for the different temperatures.

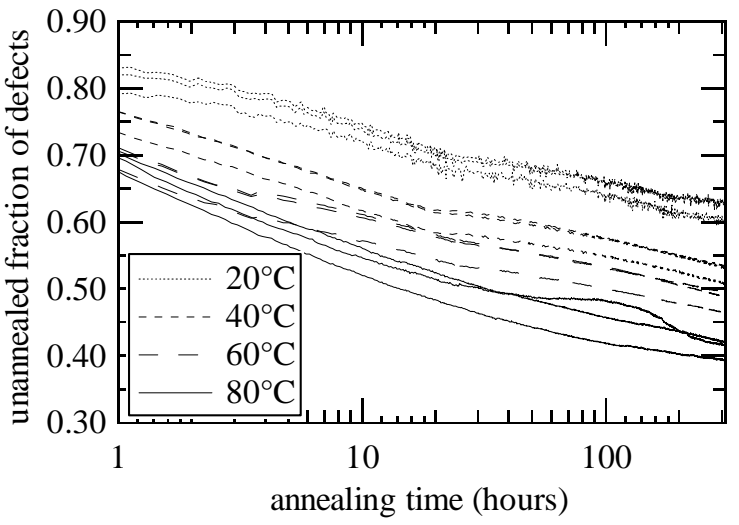

Fig. 4: Annealing of neutron damage to laser threshold current at different temperatures.

The unannealed fraction relates to the fraction of the damage remaining relative to that measured at the end of the irradiation step.

A simple model of the annealing can be constructed if we also assume that the radiation-induced defects anneal according to a first-order mechanism (exponential recovery) and that the defect lifetime $\tau$ at a given absolute temperature $T$, can be related to an activation energy $E_{a}$ by the Arrhenius formula,

$$
\tau=A \exp \left(E_{d} / k T\right)
$$


In this model the fraction of defects with a given $E_{a}$ (or time constant $\tau$ ) that remain after any given annealing time can therefore be calculated. The factor $A$, which is related to the atomic vibration frequency factor is expected to be $\sim 10^{-12} \mathrm{~s}$ for InGaAsP/InP[15]. An example of the fraction of remaining defects, for defects with a range of activation energies, is shown in Fig.5 for annealing carried out at a temperature of $20^{\circ} \mathrm{C}$.

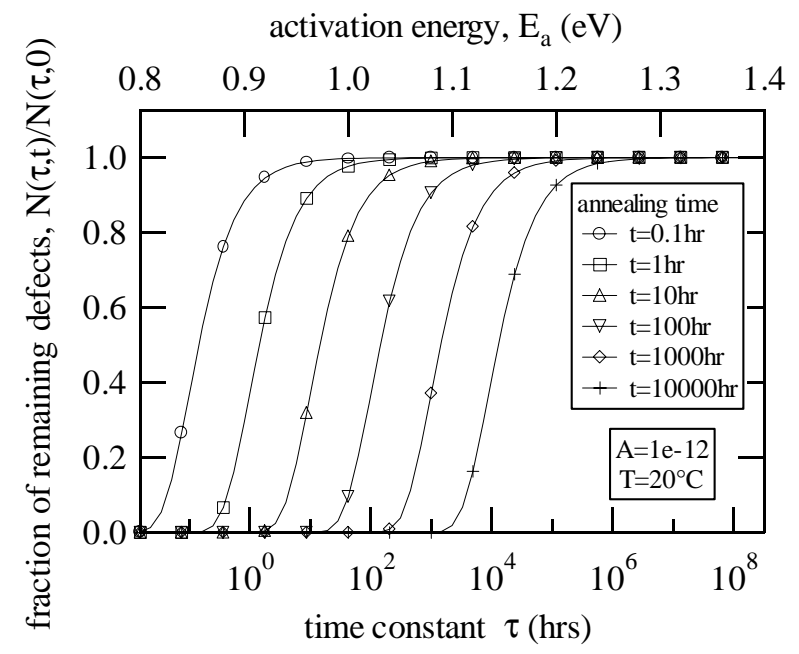

Fig. 5: Fraction of remaining defects with a given activation energy (or time constant), at different annealing times, for annealing at $20^{\circ} \mathrm{C}$.

Our previous annealing studies[6] have found that the damage recovers linearly with $\log (\operatorname{time})$. This annealing behavior is consistent with the defects having a uniform distribution of activation energies over a certain range. This simple model of the annealing was used to fit the data for the recovery at different temperatures, in order to find the range of activation energies that best described the complete set of annealing data at all 4 temperatures. To simplify the fitting procedure the annealing data was averaged for each group of 3 devices.

The model described the data best in the range of $10<\mathrm{t}(\mathrm{hrs})<310$, where there was better linearity of the measured annealing in $\log$ (time). The data and model fit are shown in Fig. 6. The activation energy spectrum that best described the data was $0.66<E_{a}<1.76 \mathrm{eV}$. The annealing observed between 20 and $80 \mathrm{C}$ actually corresponds to defects having activation energies between 1.02 and $1.40 \mathrm{eV}$. These values are similar to those measured for annealing in irradiated InGaAs photodiodes[15].

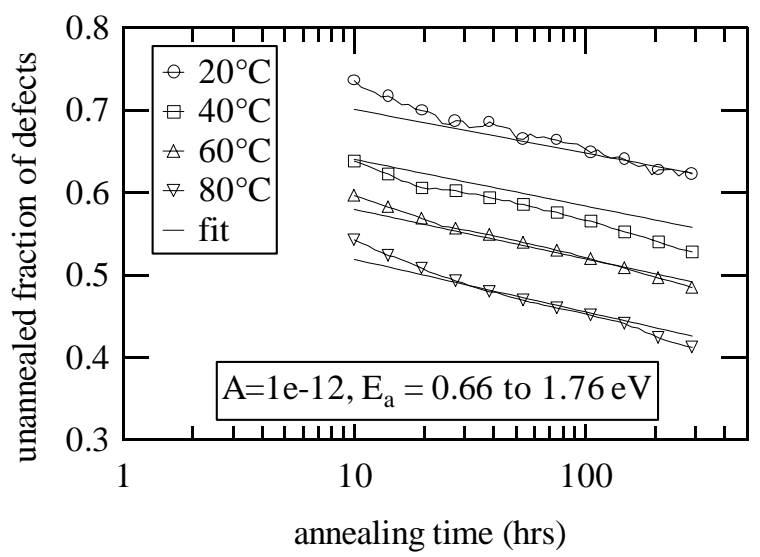

Fig. 6: Averaged annealing over $10<\mathrm{t}(\mathrm{hrs})<310$ with a fit of the annealing model described in the text. 
Clearly, the assumption of the uniform distribution of activation energies is probably too simplistic to describe correctly the full annealing characteristics. It is not possible to conclude from the data whether the defects with activation energies below $1.02 \mathrm{eV}$, or above $1.40 \mathrm{eV}$, are really uniformly distributed. Other distributions would also generate similar annealing to that measured, as long as the fraction of defects in the regions $E_{a}<1.02 \mathrm{eV}$ and $E_{a}>1.40 \mathrm{eV}$ remained the same. The fitted range also takes into account the annealing during the first 16 hours at $-13^{\circ} \mathrm{C}$ following irradiation. The fit was not very sensitive to the exact value of $A$ in that the $E_{a}$ range translated by $\pm 0.05 \mathrm{eV}$ if $A$ was changed by a factor 5 . In this analysis the weak temperature dependence of $A$ was therefore not included. The earliest data points $\left(E_{a}<1.0 \mathrm{eV}\right)$ were also not well fitted. However this could be related to the oven or, more importantly, the laser temperature not yet being completely stable.

Overall the assumption of a uniform distribution of activation energies and this particular value for $A$ is only a first step in this type of analysis. However it already gives a good description of the measured data and can be used to estimate the damage expected in lasers used in the CMS tracker optical links.

\subsection{Test $\mathrm{C}$ - Annealing vs Forward Current}

A further 8 lasers were irradiated (without electrical bias and at room temperature) in Test C. The devices were then annealed at room temperature $\left(\sim 20^{\circ} \mathrm{C}\right)$, in 4 groups of 2 devices, under forward bias conditions of $0,40,60$ and $80 \mathrm{~mA}$ respectively. The annealing data are shown in Fig. 7. Again there is a linear dependence of the annealing with $\log ($ time).

The effect of electrical bias was to accelerate the annealing, although the specific dependence of the annealing rate upon the bias current value was difficult to determine from this limited set of devices. Referring to the time-axis, the time-constants for annealing were accelerated by up to a factor of 10 by applying $80 \mathrm{~mA}$ forward bias. This resulted in an overall increase in the annealed fraction by around $18 \%$ after $100 \mathrm{hrs}$, under these particular irradiation and annealing conditions. As the annealing curves are roughly parallel, it is unlikely that this extra annealing margin will increase over longer timescales for these particular devices. Note that in a longer irradiation (to the same fluence) the acceleration factors should not be different, but after irradiation the fraction of additional defects annealing because of the bias enhancement would be larger, simply because a greater amount of annealing would have taken place during irradiation.

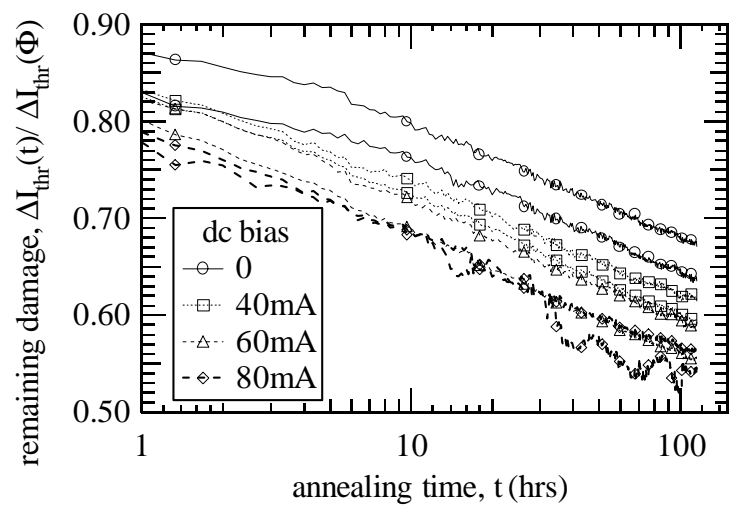

Fig. 7: Annealing of threshold current damage at different forward-bias currents following $0.8 \mathrm{MeV}$ neutron irradiation.

Acceleration of the annealing due to forward bias has been observed recently by Zhao et al.[16] in irradiated AlGaAs/GaAs lasers, where the effect was explained as being due to recombination enhanced annealing. A similar mechanism can also cause degradation in lasers, where non-radiative recombination accelerates the growth of defects[17]. Devices made of InGaAsP or InP however are not normally sensitive to this type of enhancement[17], though no systematic measurements have been made previously on irradiated InGaAsP/InP lasers. Another possible contribution to the observed acceleration is due to the heating of the device through its thermal resistance, which is $90^{\circ} \mathrm{C} / \mathrm{W}$ for these particular lasers[18]. At $80 \mathrm{~mA}$ bias the power dissipated as heat in the lasers is $\sim 90 \mathrm{~mW}$; therefore the laser junction temperature is expected to be increased by around $8^{\circ} \mathrm{C}$. This heating effect increases the rate of annealing of the defects (with $E_{a} \sim 1 \mathrm{eV}$ ) by a factor $\sim 3$ at $20^{\circ} \mathrm{C}$, explaining some of the observed acceleration. 


\section{DAMAGE AND ANNEALING INSIDE THE CMS TRACKER}

In this section we consider the long-term radiation damage that would occur if this type of lasers is used inside the CMS tracker. In Ref. [19] the method is given in more detail and the full tracker is considered. In this paper the method is only outlined briefly and we consider just the worst-case damage. This occurs for lasers situated at a radius of $22 \mathrm{~cm}$ from the beam axis, where during the first 10 years of LHC operation the radiation fluence is expected to be $1.8 \times 10^{14} / \mathrm{cm}^{2}$ high energy hadrons (mainly pions with energies between $50-1000 \mathrm{MeV}$, peaked close to $300 \mathrm{MeV}$ ). For the prediction we will assume that the damage from the full spectrum of pions is the same as that caused by the same fluence of $330 \mathrm{MeV}$ pions.

Fig. 8 illustrates the first step towards estimating the long-term damage and annealing. Using the annealing model fitted to the results of Test B, an irradiation-annealing profile resembling that in Fig. 3 was generated (modeling the irradiation and annealing in steps of 1 hour). The profile is expressed in terms of the fraction of the total radiation-induced defects that were created, with these defects having a uniform distribution of activation energies between $0.66 \mathrm{and} 1.76 \mathrm{eV}$. No additional acceleration factor for the bias current has been included as in Test B the lasers were not biased, and in the CMS tracker lasers will be biased just above threshold, therefore the bias-induced acceleration is not expected to be significant.

The peak of the damage predicted by the model in Fig. 8 can be identified approximately with the measured threshold shift of $9 \mathrm{~mA}$ in Fig.3. 100\% damage in Fig. 8 therefore corresponds to a threshold shift of 10mA when considering the effect of $0.8 \mathrm{MeV}$ neutron damage to $10^{15} \mathrm{n} / \mathrm{cm}^{2}$ within a six hour period.

The next step is then to apply the model to predict the effect expected inside the CMS tracker. Here the lasers will also be operated at $-10^{\circ} \mathrm{C}$. For devices nearest the beam-interaction point, 6 hours of LHC running at high luminosity corresponds to an irradiation of $\sim 2.9 \times 10^{10}(330 \mathrm{MeV}$ pions $) / \mathrm{cm}^{2}$. Based on the scaling factors measured in Test A, the radiation damage during this time would cause a laser threshold current increase of $3.0 \mu \mathrm{A}$.

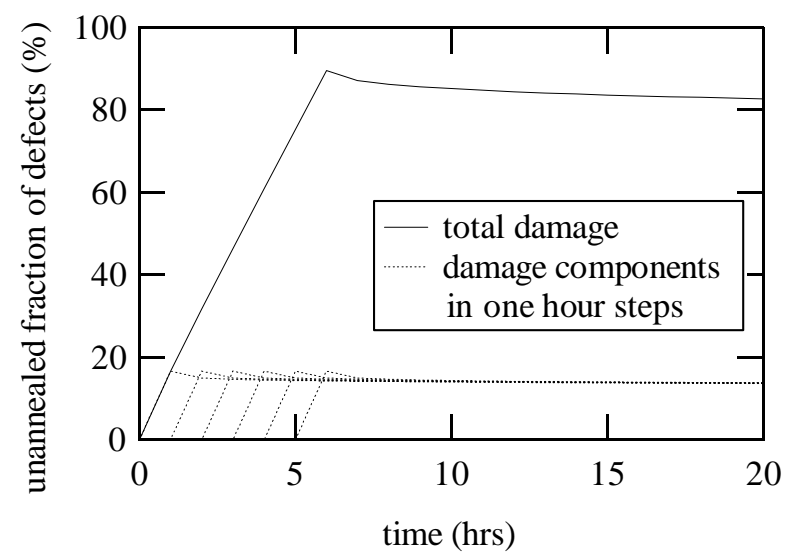

Fig. 8: Prediction of model for annealing of defects introduced during Test $\mathrm{B}$ at $-10^{\circ} \mathrm{C}$. $100 \%$ on the vertical axis corresponds to the full set of defects introduced having the activation energy range contained in the model.

Extending the model prediction to one year of LHC running gives the damage and annealing profiles in Fig. 9. An annual operation period is assumed to consist of 4000 hours with the LHC running at constant luminosity, followed by beamshutdown. These plots are again based on the fitted distribution of activation energies from Test $\mathrm{B}$, with the expectation that that the Tracker is maintained at a temperature of $-10^{\circ} \mathrm{C}$ all the time. About $22 \%$ of the total defects introduced anneal during the 4000 hours of beam time, then a further $10 \%$ of the total defects anneal during the shutdown period.

To make an estimation of the damage for the full 10 year lifetime of the tracker, the evolution of the LHC luminosity has to be taken into account. The average luminosity will be ramped up (10\%, 33\%, 67\%) over the first few years, before switching to peak conditions for the later years. The predicted damage and annealing profile over 10 years of running is 
shown in Fig. 10. (For convenience the prediction has been normalized relative to the fraction of defects that are introduced during 10 years operation with the LHC running at full luminosity.) For the worst-case of lasers situated at $22 \mathrm{~cm}$ from the beam-interaction point, the threshold increase is expected to be $14 \mathrm{~mA}$ in total.

The model predictions of the damage and annealing profile over the full 10 year lifetime should be robust since the annealing expected to occur at $-10^{\circ} \mathrm{C}$ over this timescale corresponds to the annealing actually measured in Test B (albeit at higher temperatures). The predictions therefore do not rely upon extrapolation to annealing that has not been experimentally observed.

(a) $\stackrel{\mathscr{T}}{\mathscr{E}}$

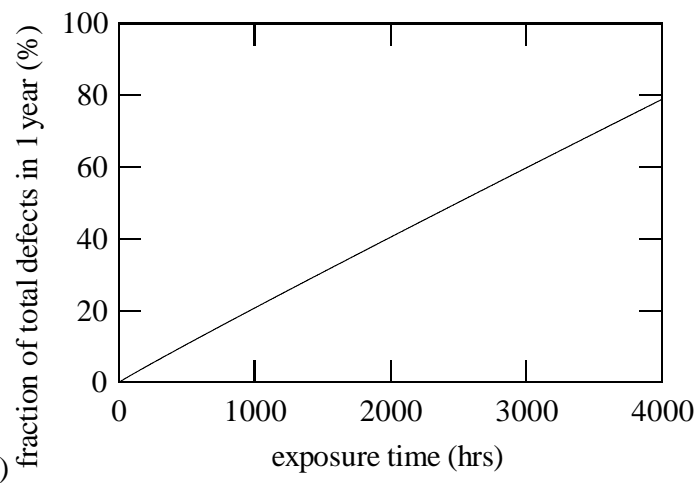

(b)

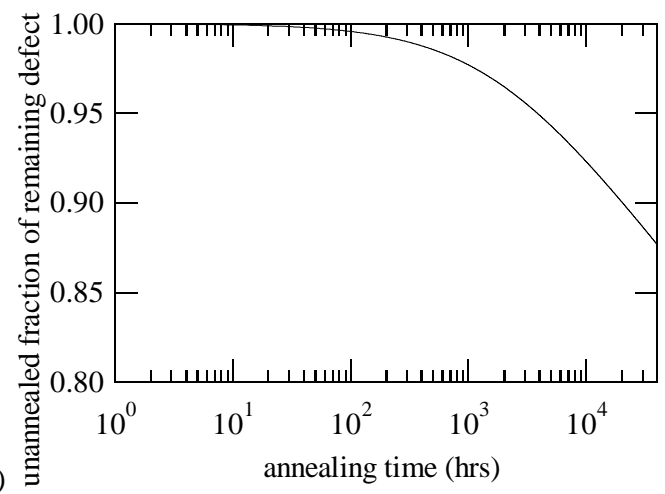

Fig. 9: Prediction of the model for defect evolution during one year of tracker operation; plot (a) shows the build up of defects during the 4000 hours of LHC running, and (b) the annealing during the LHC shut-down period.

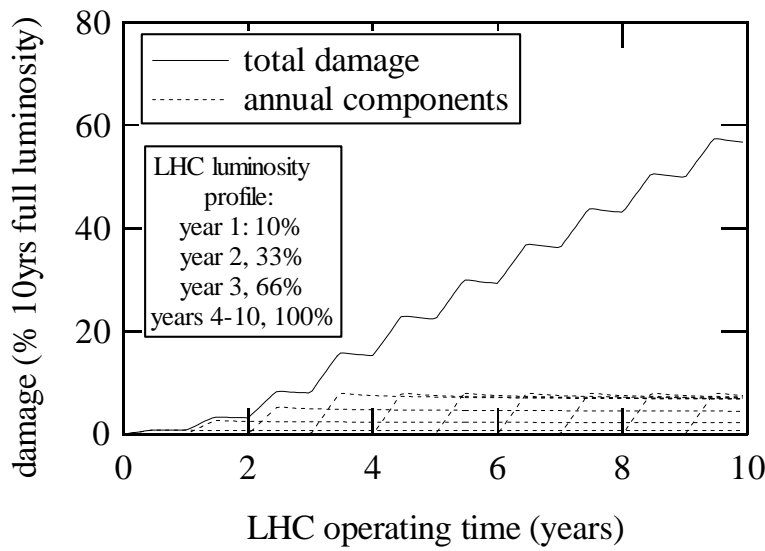

Fig. 10: Model estimation of damage evolution during full 10yr lifetime of the CMS tracker.

The damage is the fraction of that which would be introduced by 10 years of LHC running at full luminosity.

\section{CONCLUSIONS}

An extensive series of damage and annealing studies have been carried out using 1310nm InGaAsP/InP laser diodes irradiated with particles from several types of source and annealed under various conditions of temperature and electrical bias.

The displacement damage factors of $0.8 \mathrm{MeV}$ neutrons relative to $6 \mathrm{MeV}$ (average energy) neutrons, $330 \mathrm{MeV}$ pions and $24 \mathrm{GeV}$ protons were measured as $1 / 3.1,1 / 11.5$ and $1 / 8.4$ respectively, with an uncertainty of $\sim 10 \%$ in each case.

The annealing at temperatures between $20^{\circ} \mathrm{C}$ and $80^{\circ} \mathrm{C}$ has been measured in lasers irradiated to $10{ }^{15} \mathrm{n} / \mathrm{cm}^{2}$. A model predicting the annealing rate at $-10^{\circ} \mathrm{C}$ has been constructed based on the finding that the annealing behavior is generally well described by the defects having a uniform distribution of activation energies between 0.66 and $1.76 \mathrm{eV}$. 
The annealing at different forward-bias currents $(0-80 \mathrm{~mA})$ has also been measured in lasers irradiated to $10{ }^{15} \mathrm{n} / \mathrm{cm}^{2}$. The annealing rate was accelerated by up to a factor of 10 for the highest bias current, when considering the time required to anneal a given fraction of defects.

If this type of laser is used inside the CMS tracker, the increase in threshold current due to radiation damage over 10 years of operation is estimated to be $14 \mathrm{~mA}$ in the worst case of lasers situated $22 \mathrm{~cm}$ from the LHC beam axis.

\section{ACKNOWLEDGEMENTS}

We would like to thank the many people who have contributed to this long series of measurements. These include Jan Troska, Vincent Arbet Engels, and Carlos Azevedo for their involvement in the earlier irradiation tests, and Bernard Maulini, Loic Baumard and Bernard Cornet for their effort in building the various pieces of test hardware. We are also grateful to Philippe Zyromski and the staff of CEA Valduc for their valuable contribution to this work, in making the neutron irradiation and carrying out the dosimetry measurements.

\section{REFERENCES}

1. F. Vasey, V. Arbet-Engels, J. Batten, G. Cervelli, K.Gill, R. Grabit, C. Mommaert, G. Stefanini and J. Troska, "Development of Radiation-Hard Optical Links for the CMS Tracker at CERN", IEEE Trans. Nucl. Sci. Vol. 45, No. 3, p.331-337 (1998).

2. CMS Tracker Technical Design Report, CERN LHCC 98-6 (1998).

3. Addendum to CMS Tracker Technical Design Report, CERN LHCC 2000-16 (2000).

4. C.E. Barnes and J.J. Wiczer, "Radiation Effects in Optoelectronic Devices", SAND84-0771 (1984).

5. J.E. Gover and J R. Srour, "Basic Radiation Effects in Nuclear Power Electronics Technology", SAND85-0776 (1985).

6. K. Gill, V. Arbet-Engels, J. Batten, G. Cervelli, K. Gill, R. Grabit, C. Mommaert, G. Stefanini, J. Troska and F. Vasey, "Radiation Damage Studies of Optoelectronic Components for the CMS Tracker Optical Links", Proceedings of 1997 RADECS Conference, pp.405-412.

7. The gamma radiation effects were also tested to $10 \mathrm{Mrad}$ in a more recent (unpublished) study by CERN, with no significant damage in 1310nm InGaAsP/InP lasers from several manufacturers.

8. K. Gill, C. Aguilar, C. Azevedo, V. Arbert-Engels, J. Batten, G. Cervelli, R. Grabit, F. Jensen, C. Mommaert, J. Troska and F. Vasey, "Radiation damage studies of optical link components for applications in future high energy physics experiments", Proceedings SPIE Vol. 3440, p.89 (1998).

9. J. Troska, J. Batten, K. Gill and F. Vasey, "Radiation effects in commercial off-the-shelf single-mode optical fibres", Proceedings SPIE Vol. 3440, p.112 (1998).

10. J. Batten et al., Proceedings of Data Workshop, Radiations and their Effects on Devices and Systems Conference (RADECS), Cannes 1997.

11. K. Gill, C. Aguilar, V. Arbet-Engels, C. Azevedo, J. Batten, G. Cervelli, R. Grabit, F. Jensen, C. Mommaert, J. Troska and F. Vasey, "Comparative Study of Radiation Hardness of Optoelectronic Components for the CMS Tracker Optical Links", Proceedings of the 1998 RADECS Meeting, pp.67-70, September 1998.

12. P. Zyromski, "Simulating the Displacement Effect in Electronics exposed to Spatial Radiation, by Fission and $14 \mathrm{MeV}$ Neutrons", Proceedings of the conference on Radiations and their Effects on Devices and Systems (RADECS), Fontevraud 1999.

13. "Semiconductor Lasers" $2^{\text {nd }}$ Edition, G.P. Agrawal, N.K. Dutta, Van Nostrand Reinhold, New York, 1993.

14. P. W. Marshall, C. J. Dale, and E. A. Burke, "Space radiation effects on optoelectronic materials and components for a 1300nm fiber optic data bus', IEEE Trans. Nucl. Sci., Vol. 39, No. 6, pp.1982-1989 (1992).

15. G. J. Shaw, R. Walters, S. R. Messenger and G. P. Summers, "Time dependence of radiation-induced generation currents in irradiated InGaAs photodiodes", J. Appl. Phys., 74, p. 1629. (1993). (and references therein.)

16. Y.F. Zhao, R. D. Schrimpf, A. R. Patwary, M. A. Neifeld, A. W. Al-Jahoni, R. A. Weller and K. F. Galloway, "Annealing Effects on Multi-Quantum Well Laser Diodes after Proton Irradiation", IEEE Trans. Nucl. Sci., Vol. 45, No. 6, pp.2826-2832 (1998). (and references therein.)

17. "Reliability and Degradation of III-V Optical Devices" O. Ueda, Artech House, Norwood, 1996.

18. C. Aguilar. Internal CERN report on thermal resistance of lasers.

19. K. Gill et al., CMS Technical Note (in preparation). 